\title{
Clinical Value of Vascular Permeability Estimates Using Dynamic Susceptibility Contrast MRI: Improved Diagnostic Performance in Distinguishing Hypervascular Primary CNS Lymphoma from Glioblastoma
}

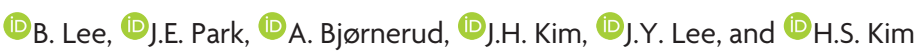

\begin{abstract}
BACKGROUND AND PURPOSE: A small subset of primary central nervous system lymphomas exhibits high cerebral blood volume, which is indistinguishable from that in glioblastoma on dynamic susceptibility contrast MR imaging. Our study aimed to test whether estimates of combined perfusion and vascular permeability metrics derived from DSC-MR imaging can improve the diagnostic performance in differentiating hypervascular primary central nervous system lymphoma from glioblastoma.
\end{abstract}

MATERIALS AND METHODS: A total of 119 patients (with 30 primary central nervous system lymphomas and 89 glioblastomas) exhibited hypervascular foci using the reference method of leakage-corrected CBV (reference-normalized CBV). An alternative postprocessing method used the tissue residue function to calculate vascular permeability (extraction fraction), leakage-corrected CBV, cerebral blood flow, and mean transit time. Parameters were compared using Mann-Whitney $U$ tests, and the diagnostic performance to distinguish primary central nervous system lymphoma from glioblastoma was calculated using the area under the curve from the receiver operating characteristic curve and was cross-validated with bootstrapping.

RESULTS: Hypervascular primary central nervous system lymphoma showed similar leakage-corrected normalized CBV and leakagecorrected CBV compared with glioblastoma $(P>.05)$; however, primary central nervous system lymphoma exhibited a significantly higher extraction fraction $(P<.001)$ and $\mathrm{CBF}(P=.01)$ and shorter MTT $(P<.001)$ than glioblastoma. The extraction fraction showed the highest diagnostic performance (the area under the receiver operating characteristic curve [AUC], 0.78; 95\% confidence interval, $0.69-0.85$ ) for distinguishing hypervascular primary central nervous system lymphoma from glioblastoma, with a significantly higher performance than both CBV (AUC, 0.53-0.59, largest $P=.02$ ) and CBF (AUC, 0.72) and MTT (AUC, 0.71).

CONCLUSIONS: Estimation of vascular permeability with DSC-MR imaging further characterizes hypervascular primary central nervous system lymphoma and improves diagnostic performance in glioblastoma differentiation.

ABBREVIATIONS: $\mathrm{AUC}=$ area under the receiver operating characteristic curve; $\mathrm{CBV}_{\text {res }}=$ leakage-corrected $\mathrm{CBV}$ derived using the proposed residue functionbased correction method; DCE $=$ dynamic contrast-enhanced; $\mathrm{EF}=$ extraction fraction; $K^{\text {trans }}=$ contrast agent transfer constant; $n C B V_{\text {ref }}=$ leakage-corrected normalized CBV derived using the reference-correction method; PCNSL = primary central nervous system lymphoma

A common manifestation in primary central nervous system lymphoma (PCNSL) on dynamic susceptibility contrast MR imaging is low cerebral blood volume, ${ }^{1-6}$ which distinguishes PCNSL from glioblastoma with a diagnostic accuracy as high as $90.9 \% .^{3}$ This can be explained by less prominent neovasculariza-

Received January 24, 2018; accepted after revision May 1.

From the Department of Radiology (B.L.), Seoul Metropolitan Government-Seoul National University, Boramae Medical Center, Seoul, Korea; Department of Radiology and Research Institute of Radiology (J.E.P., H.S.K.), University of Ulsan College of Medicine, Asan Medical Center, Seoul, Korea; Department of Diagnostic Physics (A.B.), Rikshopitalet University Hospital, Oslo, Norway; NordicNeuroLab (J.H.K.), Seoul, Korea; and Department of Radiology (J.Y.L.), Soonchunhyang University Bucheon Hospital, Bucheon, Korea.

This research was supported by the National Research Foundation of Korea grant funded by the Korean government (Ministry of Science, ICT and Future Planning) (grant number: NRF-2017RIC1B2007258) and by a grant from the National R\&D Program for Cancer Control, Ministry of Health and Welfare, Republic of Korea tion and higher vascular permeability in histopathologic specimens compared with glioblastoma. ${ }^{7,8}$ Also, low cerebral blood volume $(\mathrm{CBV})$ can be explained by the signal response in DSC-MR imaging, in which the T1-shortening effect in interstitial tissue from the extravasated contrast agent can overwhelm the $\mathrm{T}^{\star}$ effect, thereby reducing apparent $\mathrm{CBV}^{9,10}$ in PCNSLs if leakage-correction is not applied. Moreover, a small subset of PCNSLs may exhibit high CBV, comparable with the CBV of high-grade

\footnotetext{
(1720030). The funders had no role in the study design, data collection and analysis, decision to publish, or preparation of the article.

Please address correspondence to Ji Eun Park, MD, PhD, Department of Radiology and Research Institute of Radiology, University of Ulsan College of Medicine, Asan Medical Center, 43 Olympic-ro 88, Songpa-Gu, Seoul 05505, Korea; e-mail: jieunp@gmail.com

- Indicates open access to non-subscribers at www.ajnr.org

http://dx.doi.org/10.3174/ajnr.A5732
} 
gliomas $^{11,12}$; this so-called hypervascular PCNSL thus becomes indistinguishable from glioblastoma using CBV.

Among the important vascular parameters of tumor vasculature and vessel permeability, PCNSL exhibits a distinctive difference in vascular permeability compared with glioblastoma. The T1-dominant leakage has been shown using dynamic contrastenhanced (DCE) MR imaging in that substantial blood-brain barrier leakage and higher contrast agent transfer constant $\left(K^{\text {trans }}\right)$ and $\mathrm{K}_{\mathrm{ep}}$ (flux rate constant) values ${ }^{7}$ were observed in PCNSL than in glioblastoma. Histopathologic evaluation revealed intact vascular integrity in glioblastoma despite endothelial proliferation, whereas the PCNSL exhibited destroyed vessel architecture, thereby leading to differences in DCE parameters. Meanwhile, glioblastoma shows a dominant $\mathrm{T} 2{ }^{\star}$ effect from rapid and abundant accumulation of contrast in the interstitial space, which is known to be greater than the T1-shortening effect. ${ }^{7,13}$ The addition of DCE-MR imaging could thus potentially aid in the differentiation of hypervascular PCNSL from glioblastoma. However, the addition of this technique in addition to DSC-MR imaging would require a double contrast agent injection and additional complex image processing steps. An alternative strategy is therefore to derive both perfusion- and permeability-related metrics from the single DSC-MR imaging acquisition.

A combined perfusion/permeability analysis method by DSC-MR imaging was recently proposed by Bjørnerud et al. ${ }^{13}$ In this approach, both perfusion and permeability metrics are obtained by fitting appropriate kinetic models to the tissue residue function obtained by deconvolution with an automatically derived arterial input function. ${ }^{14,15}$ In addition to providing estimates of cerebral blood flow, mean transit time (MTT), and the extraction fraction (EF), the method also provides leakage-corrected CBV values, which are unaffected by variations in MTT. ${ }^{14}$ Given the substantial contrast agent leakage in both glioblastoma and PCNSL, CBV needs to be leakage-corrected, and the most established method of correction is the approach first proposed by Weisskoff et $\mathrm{al}^{16}$ and later tested for clinical performance by Boxerman et al. ${ }^{10}$ In this reference-correction method, extravasation is estimated by voxelwise deviation from a nonleaky reference tissue-response curve. The method is thus inherently sensitive to deviations in tumor mean transit time compared with the reference tissue. ${ }^{13}$ The alternative approach tested here is therefore hypothesized to have advantages over the reference method in that it provides additional perfusion- and extraction-related parameters and a more unbiased estimate of leakage-corrected CBV.

Thus, the purpose of our study was to test whether estimates of combined perfusion and permeability metrics obtained from DSC-MR imaging using the proposed method improve diagnostic performance in differentiating hypervascular PCNSL from glioblastoma.

\section{MATERIALS AND METHODS \\ Patients}

Our institutional (Asan Medical Center, Seoul, Korea) review board approved this retrospective study, and the requirement for informed consent was waived. From November 2006 to December 2016, we retrospectively reviewed patients from the data base of our institution who were diagnosed with pathologically proved PCNSL ( $n=363$ ) and underwent DSC imaging before treatment. Inclusion criteria for PCNSL were as follows: 1) no prior history of chemotherapy, radiation therapy, or corticosteroid treatment; and 2) appropriate DSC imaging quality. Then, 2 experienced neuroradiologists (J.Y.L. and J.E.P.), who did not participate in any other image review, independently determined the vascularity of PCNSL based on the CBV maps, as visualized in the PACS, which were processed using the reference method of Weisskoff et $\mathrm{al}^{16}$ and Boxerman et $\mathrm{al}^{10}$ as part of the routine workflow. In case of a discrepancy, they discussed the issue with a senior radiologist (H.S.K., with 18 years of experience) to reach a consensus. Hypervascular PCNSLs were defined as showing higher CBV foci in the solid-enhancing tumor portion compared with the contralateral cortex. Among 363 patients with PCNSL, 30 patients (16 men; median age, 61.9 years; range, 56.5-67.3 years) were selected. As a comparison, a consecutive enrollment of patients with pathologically proved, newly diagnosed glioblastoma $(n=$ 105) was performed using the radiologic data base from March 2015 to November 2016. The same criterion of visual assessment for hypervascular foci was applied, and 89 patients (54 men; mean age, 55.9 years of age; age range, 53.7-58.2 years) with glioblastoma were included in the study.

\section{MR Imaging}

All MR imaging studies were performed on a 3T unit (Achieva; Philips Healthcare, Best, the Netherlands) using an 8-channel head coil. The brain tumor imaging protocol consisted of the following sequences: T1-weighted, T2-weighted, fluid attenuated inversion recovery, diffusion-weighted, contrast-enhanced T1weighted, and DSC-MR imaging.

A contrast-enhanced high-resolution anatomic $3 \mathrm{D}$ volume image was obtained using a gradient-echo T1-weighted sequence with the following parameters: TR/TE, 9.8/4.6 ms; flip angle, $10^{\circ}$; FOV, $256 \mathrm{~mm}$; matrix, $512 \times 512$; and slice thickness, $1 \mathrm{~mm}$ with no gap. Then, DSC-MR imaging was performed using a gradientecho, echo-planar sequence during the injection of a standard dose of $0.1 \mathrm{mmol} / \mathrm{kg}$ of intravenous gadoterate meglumine (Dotarem; Guerbet, Aulnay-sous-Bois, France) at a rate of $4 \mathrm{~mL} / \mathrm{s}$. The bolus injection of contrast material was followed by a $20-\mathrm{mL}$ bolus injection of saline at the same injection rate. The DSC-MR imaging parameters were as follows: TR/TE, 1808/40 ms; flip angle, 35ㅇ FOV, $24 \mathrm{~cm}$; slice thickness/gap, 5/2 mm; matrix, $128 \times$ 128; total acquisition time, 1 minute 54 seconds. Subsequently, a postcontrast T1-weighted 3D gradient-echo sequence was acquired with following parameters: TR/TE, 9.8/4.6 ms; flip angle, $8^{\circ}$; matrix, $512 \times 512$; slice thickness, $3 \mathrm{~mm}$; FOV , 22-26 cm.

\section{Image Postprocessing}

DSC-MR imaging data were transferred to an independent workstation and processed using nordicICE (Version 4.0.6; NordicNeuroLab, Bergen, Norway) software. The postprocessing method is shown in Fig 1.

First, the reference method of Weisskoff et $\mathrm{al}^{16}$ and Boxerman et $\mathrm{al}^{10}$ was applied to calculate leakage-corrected CBV. Here, deconvolution is performed with respect to a "mean tissue curve," which is assumed to be unaffected by extravasation; the mean 


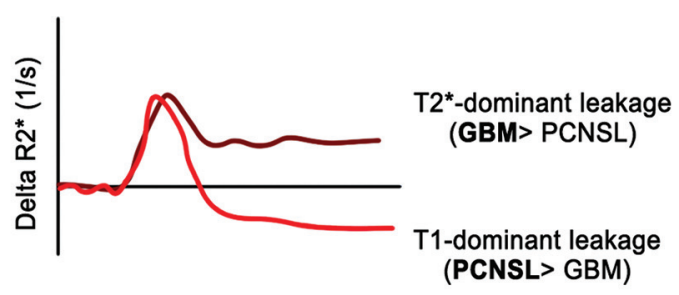

Leakage correction method

(PCNSL> GBM)

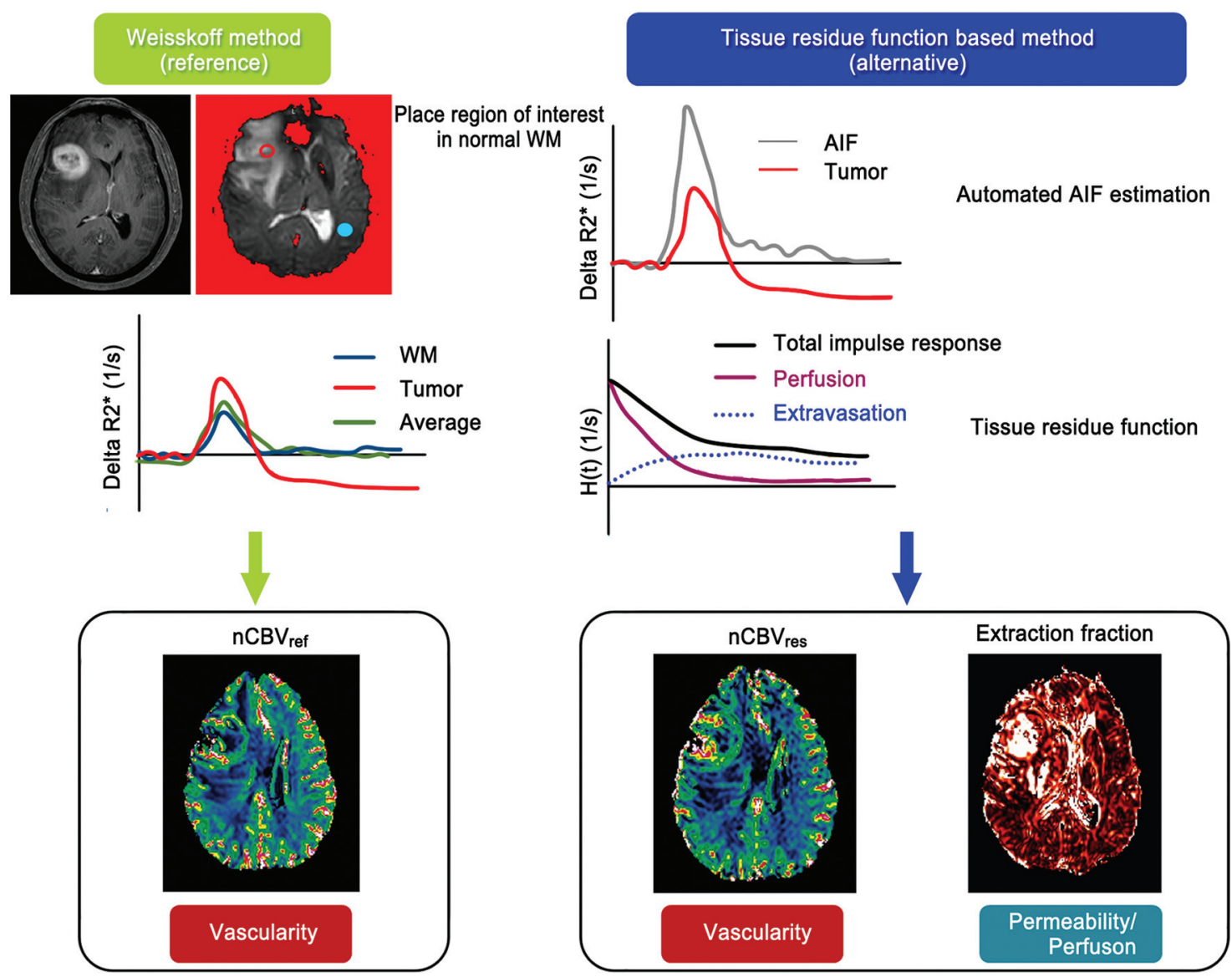

FIG 1. Workflow of the 2 presented analysis approaches: the reference Weisskoff method and the alternative tissue residue function-based method. In the reference method, an ROI is placed on the normal-appearing white matter and normalized cerebral blood volume is calculated. In the alternative method, both arterial input function and tissue residue function are estimated. The tissue residue function comprises both apparent tissue extravasation and a perfusion component. Then, leakage-corrected cerebral blood volume, reflecting tissue perfusion, and extraction fraction, reflecting the ratio of permeability/perfusion, are calculated. AIF indicates arterial input function; GBM, glioblastoma multiforme; R2*, gradient echo relaxivity.

transit times of the reference tissue and leakage-corrected tissues are assumed to be identical. For CBV calculation, the dynamic signal-intensity curve was converted to a time-relaxation rate curve, and relative CBV was estimated from the area under the time-relaxation rate curve. ${ }^{13}$ Pixel-wise relative $\mathrm{CBV}$ values were then normalized relative to the mean value of the ROI placed on the contralateral normal-appearing white matter (size range, $\left.30-50 \mathrm{~mm}^{2}\right)$, resulting in a normalized CBV map $\left(\mathrm{nCBV}_{\text {ref }}\right)$. This process was performed in a fully automated batch mode, and motion-correction was applied before analysis.

Second, an alternative method, here called the residue function-based contrast leakage-correction method, was applied. ${ }^{13}$ This method is based on fitting of the tissue residue function to the 2-compartment uptake kinetic model. ${ }^{17}$ This provides a closed functional description of the resulting leakage-affected tis- sue residue function. ${ }^{14}$ The general equation for the relationship between arterial input function and tissue response is then given by

1)

$$
C_{\mathrm{t}}(t)=F \times C_{\mathrm{a}}(t) \otimes H(t),
$$

where $C_{\mathrm{t}}(t)$ and $C_{\mathrm{a}}(t)$ are the tissue response and arterial input functions, respectively, $\otimes$ is the convolution operator, and $F$ is fractional tissue perfusion. $H(t)$ describes the probability of a tracer being present in a tissue at time $t$ following an instantaneous tracer bolus. The product $F \times H(t)=R(t)$ can be estimated using standard deconvolution techniques, and $F$ is then obtained from the peak height of the resulting $R(t) .{ }^{13}$ The 2-compartment uptake model accounts for continuous extravasation along the length of the capillaries but assumes negligible reflux of the contrast agent from the extracellular extravascular space to the 
plasma space during the observation period. ${ }^{17}$ Then the residue function can be expressed by

$$
\text { 2) } \quad H(t)=\exp \left(-\frac{t}{T_{\mathrm{p}}}\right)+E_{\mathrm{x}}\left[1-\exp \left(-\frac{t}{T_{\mathrm{p}}}\right)\right] \text {, }
$$

where $T_{\mathrm{p}}$ is the plasma mean transit time and $E_{\mathrm{x}}$ is the extraction fraction describing the fraction of contrast agent that is extracted during the first passage through tissue. $E_{\mathrm{x}}$ can be expressed as the ratio

3)

$$
E_{\mathrm{x}}=K^{\mathrm{trans}} / F \text {, }
$$

where $K^{\text {trans }}$ is the contrast agent transfer constant. $H(t)$ estimated from deconvolution was then fitted to Equation 2 using a nonlinear least-squares algorithm, giving $T_{\mathrm{p}}$ and $E_{\mathrm{x}}$.

The capillary mean transit time is related to the time constant $T_{\mathrm{p}}$ according to

$$
\operatorname{MTT}=\frac{T_{\mathrm{p}}}{1-E_{\mathrm{x}}} .
$$

From the central volume principle, the tissue volume fraction is given by $V=F \times M T T$, and capillary blood volume fraction (independent of extravasation) is thus given by

$$
C B V_{\text {res }}=\varsigma \times F \frac{T_{\mathrm{p}}}{1-E_{\mathrm{x}}},
$$

where $s$ is a scaling factor (including tissue density and time unit conversion) and $F$ is fractional tissue perfusion as defined in Equation 1, from the peak height of the residue function, as obtained from deconvolution using singular-value decomposition. ${ }^{13}$ Because leakage-corrected $\mathrm{CBV}\left(\mathrm{CBV}_{\text {res }}\right)$ is calculated by deconvolution with (and automatically determined by) an arterial input function, no further normalization was applied here. ${ }^{13}$

\section{Image Analysis}

Among the DSC parameters, the parameter that relates to vascular permeability was the extraction fraction, which describes the fraction of the contrast agent that is extracted during the first passage through the tissue. We hypothesized that the EF can show discriminatory power in hypervascular PCNSL showing high CBV. Also, other macroscopic perfusion parameters of CBV, CBF, and MTT were analyzed. All parametric maps, $\mathrm{nCBV}_{\text {ref, }} \mathrm{CBV}_{\text {res, }} \mathrm{MTT}$, and EF, were coregistered to the contrast-enhanced $\mathrm{T} 1$ images. The volume of interest was drawn section by section on the contrastenhancing T1-weighted images using the semiautomated segmentation method with the nordicICE software. This includes all contrastenhancing areas, excluding the vessels and necrosis. The VOI was drawn by a neuroradiologist (B.E.L., with 2 years of experience in neuroradiology) and confirmed by a senior radiologist (J.E.P., with 5 years of experience) to ensure proper segmentation. The overall value of each VOI in DSC parameters was obtained automatically with the software by summing up all values from each axial slice, which was then averaged.

\section{Statistical Analysis}

Clinical characteristics of patients with hypervascular PCNSLs and glioblastoma were compared with the $\chi^{2}$ test. Values are expressed as mean \pm SD for continuous variables, and all continu-
Table 1: Clinical characteristics of the study patients ${ }^{a}$

\begin{tabular}{lccc}
\hline & $\begin{array}{c}\text { PCNSL } \\
(\boldsymbol{n}=\mathbf{3 0})\end{array}$ & $\begin{array}{c}\text { Glioblastoma } \\
(\boldsymbol{n}=\mathbf{8 9})\end{array}$ & $\begin{array}{c}\boldsymbol{P} \\
\text { Value }\end{array}$ \\
\hline No. of male patients & $16(46.7 \%)$ & $54(60.7 \%)$ & .48 \\
Age $(\mathrm{yr})$ & $61.9 \pm 2.6$ & $55.9 \pm 10.8$ & .04 \\
Tumor volume $(\mathrm{mL})$ & $14.9 \pm 14.5$ & $13.9 \pm 11.4$ & .70 \\
\hline
\end{tabular}

a Data are expressed as means for continuous variables. Numbers in parentheses are percentages.

Table 2: Comparisons of dynamic susceptibility contrast imaging parameters between hypervascular PCNSLs and glioblastomas ${ }^{\mathrm{a}}$

\begin{tabular}{lccc}
\multicolumn{1}{c}{ Parameter } & $\begin{array}{c}\text { Hypervascular } \\
\text { PCNSL }\end{array}$ & Glioblastoma & P Value \\
\hline EF $(\%)$ & $7.88 \pm 4.31$ & $3.87 \pm 1.46$ & $<.001^{\mathrm{b}}$ \\
$\mathrm{CBV}_{\text {res }}$ (relative units) & $2.85 \pm 1.32$ & $2.73 \pm 1.10$ & .44 \\
$\mathrm{MTT}$ (sec) & $5.77 \pm 1.85$ & $7.40 \pm 2.22$ & $<.001^{\mathrm{b}}$ \\
rCBF (relative units) & $2.88 \pm 1.24$ & $2.10 \pm 1.32$ & $.01^{\mathrm{b}}$ \\
nCBV $_{\text {ref }}$ (relative units) & $2.67 \pm 1.15$ & $2.93 \pm 0.99$ & .14 \\
\hline
\end{tabular}

Note:- $r$ CBF indicates relative CBF.

a Data are means.

b Significant.

ous variables were assessed for normality using the Shapiro-Wilk test. Because continuous variables showed unequal variances and unequal sample sizes, the Mann-Whitney $U$ test was performed to compare DSC parameters between hypervascular PCNSLs and glioblastoma.

Diagnostic performance to distinguish hypervascular PCNSLs from glioblastomas was calculated using receiver operating characteristic curve analysis. For receiver operating characteristic curve analysis, optimal thresholds of the imaging parameters were determined by maximizing the Youden index (sum of the sensitivity and 1 -specificity values), ${ }^{18}$ which was calculated to differentiate the 2 entities. Diagnostic performance of the DSC parameters was further validated using leave-one-out cross-validation with 100 -fold bootstrapping. This procedure assigns 1 subject as the validating set and the other subjects as the training set and repeats the calculation until every case has been selected as the validation set, with replacement allowed. For the comparison of the areas under the curve (AUCs) and cross-validation with bootstrap resampling, the pROC and cvTools packages in R, Version 3.3.3 (http://www.r-project.org) were used. $P$ values $<.05$ indicated a significant difference.

\section{RESULTS}

Patient characteristics are summarized in Table 1. Patients with hypervascular PCNSL were older than those with glioblastoma (61.9 years versus 55.9 years, $P=.04$ ). There was no significant difference in tumor volume between the 2 groups.

\section{Comparison of DSC Parameters between Hypervascular PCNSL and Glioblastoma}

Table 2 summarizes the results of DSC parameter comparison. Hypervascular PCNSLs demonstrated significantly higher EF $(7.88 \% \pm 4.31 \%)$ compared with glioblastomas $(3.87 \% \pm 1.46 \%$, $P<.001)$. For CBV, there was not a significant difference between hypervascular PCNSLs and glioblastomas for either $\mathrm{nCBV}_{\text {ref }}$ $(2.67 \pm 1.15$ versus $2.93 \pm 0.99 ; P=.14)$ or $\mathrm{CBV}_{\text {res }}$ (mean, $2.85 \pm$ 1.32 versus $2.73 \pm 1.10 ; P=.44$ ).

Also, hypervascular PCNSLs showed shorter MTT (5.77 \pm 1.85 seconds $)$ and higher relative CBF $(2.88 \pm 1.24)$ compared 


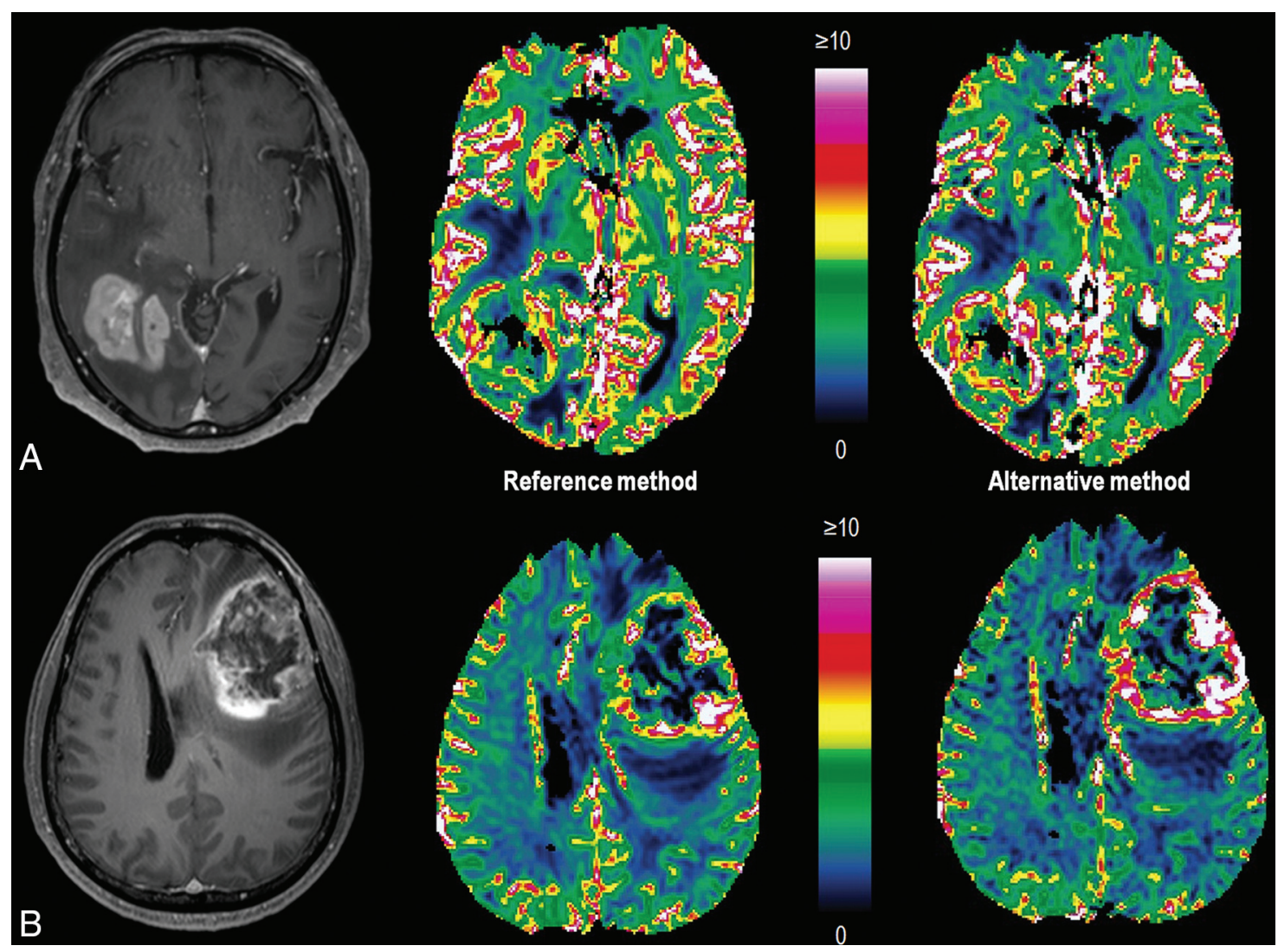

FIG 2. Comparison of cerebral blood volume between hypervascular PCNSL and glioblastoma. A, PCNSL shows homogeneous contrast enhancement and increased CBV in the right parietotemporal lobe. Note that the CBV is slightly increased in the alternative method (CBV res) (right) compared with the reference method $\left(\mathrm{nCBV}_{\text {ref }}\right)($ left). $B$, Glioblastoma shows heterogeneous enhancement and increased $\mathrm{CBV}$ in the left frontal lobe. Note that the CBV is similar with both methods.

with glioblastomas $(\mathrm{MTT}, 7.40 \pm 2.22$ seconds; $P<.001$; and relative $\mathrm{CBF}, 2.10 \pm 1.32, P<0.01)$.

Figures 2 and 3 show representative DSC parameters from the patients with hypervascular PCNSL and glioblastoma.

\section{Comparison of Diagnostic Performance Using DSC Parameters to Distinguish PCNSL from Glioblastoma}

Among the DSC-MR imaging parameters tested, EF showed the highest diagnostic performance (AUC, 0.78; 95\% confidence interval, 0.6-0.85) for differentiating hypervascular PCNSL from glioblastoma. The optimal threshold for EF was 6.25, with a sensitivity of $70.0 \%$, specificity of $89.9 \%$, and diagnostic accuracy of $84.9 \%$ (Table 3).

Diagnostic performances using CBV were generally poor, both for $\mathrm{CBV}_{\text {res }}$ (AUC, 0.53 ; 95\% CI, 0.43-0.62) and $\mathrm{nCBV}_{\text {ref }}$ (AUC, 0.59 ; 95\% CI, 0.50-0.68) with EF significantly improving diagnostic performance compared with both $\mathrm{CBV}_{\text {res }}(P=.002)$ and $\mathrm{nCBV}_{\text {ref }}(P=.02)$.

Moreover, EF indicated better diagnostic performance compared with MTT (AUC, 0.71; 95\% CI, 0.62-0.79) and relative CBF (AUC, 0.72; 95\% CI, 0.63-0.79), but these differences were not statistically significant.

The leave-one-out cross-validation demonstrated the same trend, in which EF showed the highest diagnostic performance in distinguishing hypervascular PCNSL from glioblastoma.

\section{DISCUSSION}

In this proof-of-concept study, we have applied a novel analysis approach to estimate both perfusion- and permeability-related metrics from a single DSC-MR imaging acquisition. About $8.3 \%$ of PCNSLs exhibited hypervascularity in our patient population, with comparable or higher $\mathrm{CBV}$ than in glioblastomas on DSC-MR imaging. The conditions of these patients would be indistinguishable with conventional processing methods. The main finding of the study is that estimation of the contrast agent extraction fraction using this approach can aid in the differentiation of hypervascular PCNSLs from glioblastomas. The EF showed higher diagnostic performance in differentiating hypervascular PCNSL from glioblastoma compared with all other metrics investigated (leakage-corrected CBV, MTT, and CBF). Thus, estimates of vascular permeability from DSC-MR imaging have clinical value to further characterize hypervascular PCNSLs and improve glioblastoma differentiation.

Previous DCE-MR imaging studies have shown increased vascular permeability, reflected in higher $K^{\text {trans }}$ in PCNSLs, suggesting a greater degree of blood-brain barrier breakdown. ${ }^{6,7}$ Poor neovascularization and angiocentric growth patterns in PCNSL compared with glioblastoma result in increased permeability ${ }^{19,20}$ and support the observed increased EF in hypervascular PCNSL. Because the extraction fraction reflects the ratio of permeability to flow, the measured elevated EF in hypervascular PCNSL com- 


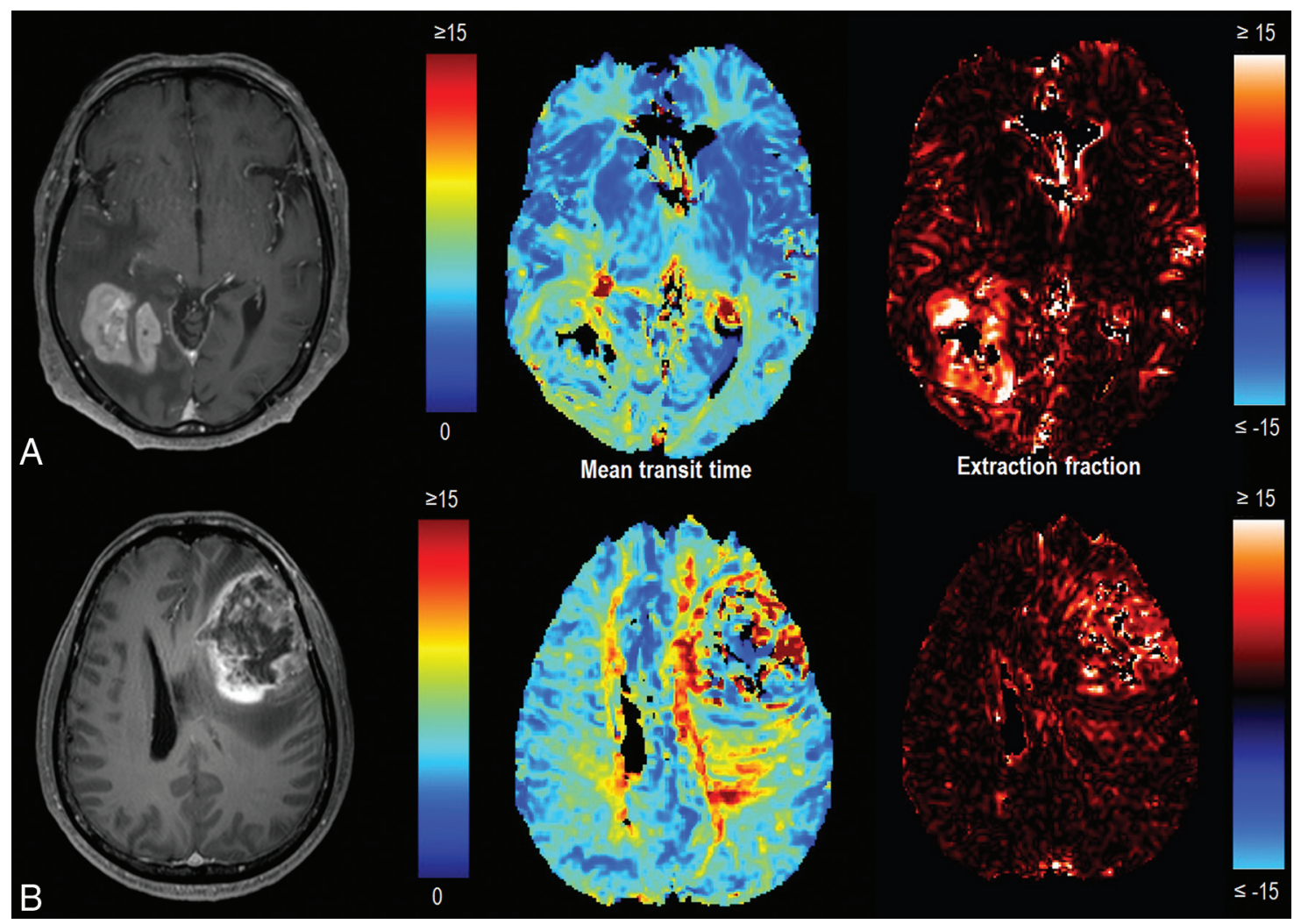

FIG 3. Comparison of the extraction fraction and mean transit time between hypervascular PCNSL and glioblastoma. A, PCNSL shows low MTT and a high extraction fraction. $B$, Glioblastoma shows high MTT and a low extraction fraction. There are distinct differences in the EF and MTT between the 2 tumors.

Table 3: Comparison of diagnostic performance among DSC parameters in distinguishing hypervascular PCNSL from glioblastoma

\begin{tabular}{lccccc}
\hline Parameter & AUC & 95\% Cl & $\begin{array}{c}\text { Standard } \\
\text { Error }\end{array}$ & $\begin{array}{c}\text { Optimal } \\
\text { Threshold }\end{array}$ & $\begin{array}{c}\text { Cross-Validated } \\
\text { AUC }\end{array}$ \\
\hline EF & 0.78 & $0.69-0.85$ & 0.06 & 6.25 & 0.76 \\
CBV $_{\text {res }}$ & 0.53 & $0.43-0.62$ & 0.06 & 3.71 & 0.49 \\
MTT & 0.71 & $0.62-0.79$ & 0.05 & 6.81 & 0.73 \\
rCBF & 0.72 & $0.63-0.79$ & 0.05 & 1.86 & 0.72 \\
nCBV $_{\text {ref }}$ & 0.59 & $0.50-0.68$ & 0.06 & 1.90 & 0.55 \\
\hline
\end{tabular}

Note:- $-\mathrm{rCBF}$ indicates relative $\mathrm{CBF}$.

pared with glioblastoma suggests that permeability increases relatively more than perfusion in these tumors. $\mathrm{EF}$ is $<10 \%$ in both tumor types, suggesting that $K^{\text {trans }}$ is a permeability-limited parameter and not perfusion-limited in both gliomas and PCNSLs. $^{21}$

The alternative method separates $\mathrm{T} 1$ - or $\mathrm{T} 2$ - $^{\star}$ dominant leakage using the tissue residue function, whereby T1-dominant leakage results in a negative tail and $\mathrm{T} 2^{*}$-dominant leakage results in a positive tail ${ }^{13,14}$ in the residue function. The reference method was originally developed to only correct for T1-dominant leakage, but a modified version of the algorithm was used here, which also corrects for $\mathrm{T} 2{ }^{\star}$-dominant leakage. ${ }^{13}$ Regardless of the correction approach used, 1 inherent limitation of using DSC-MR imaging to derive permeability parameters is that the dominant relaxation effect following contrast agent extravasation is poorly defined and unpredictable. If a combination of $\mathrm{T} 1$ and $\mathrm{T} 2{ }^{\star}$ effects are present, then permeability will be underestimated because the 2 relaxation effects will have an opposing influence on the magnitude of the resulting permeability parameters. To obtain correct permeability estimates from DSC-MR imaging, one would therefore ideally like to completely eliminate $\mathrm{T} 1$ effects following extravasation. Previous studies have shown that $\mathrm{T} 1$ effects can be reduced by injecting a small prebolus contrast agent to saturate T1 effects or by reducing the flip angle of the DSC sequence. ${ }^{22}$

In the current study, a small flip angle $\left(35^{\circ}\right)$ method was used to reduce the sensitivity to the $\mathrm{T} 1$ leakage effect compared with the large flip angle T1-sensitive method. On the other hand, a preload bolus method is a widely accepted protocol to minimize the initial tissue T1 effects; the only concern for a prebolus method is a residual baseline level of contrast agent with a $2^{*}$ effect, ${ }^{23}$ which may increase relative CBV. ${ }^{24}$ Along with permeability differences, hypervascular PCNSLs demonstrated shorter MTT and higher $\mathrm{CBF}$ compared with glioblastomas. These findings differ from those in previous studies, which either demonstrated no difference in CBF between PCNSL and high-grade glioma ${ }^{25}$ or higher $\mathrm{CBF}$ in glioblastoma compared with PCNSL. ${ }^{26}$ This difference may come from a unique population of hypervascular PCNSLs because most manifest a lower CBV than glioblastomas.

Our results further illustrate the inherent biologic differences in PCNSL relative to glioblastoma; however, hypervascular PCNSL presents as tumor infiltration around pre-existing vessels, which may result in lower MTT and higher CBF compared with glioblastoma. ${ }^{8}$ Conversely, glioblastomas with heterogeneous microvascular density may result in increased MTT. ${ }^{27}$ Higher MTT and lower CBF in glioblastomas can be explained by previous pathologic studies revealing the chaotic architecture of the neovessels with arteriovenular shunts and compromised capillary 
paths ${ }^{28}$ that increase capillary transit time and decrease tissue perfusion $^{29}$ in the contrast-enhancing tumor portion. These hemodynamic parameters are difficult to obtain from a reference leakage-correction method ${ }^{16}$ because the model assumes that mean transit time and bolus arrival time are the same in tumor and normal tissues. $^{13,15}$

Our study is limited in that it used a small amount of patient data, especially regarding hypervascular PCNSLs. Second, direct correlations are lacking between perfusion parameters and histologic features such as microvessel density and endothelial ultrastructure. Third, permeability parameters and the relative contributions of $\mathrm{T} 1$ - versus $\mathrm{T} 2{ }^{*}$-dominant leakage effects in the different tumor subgroups should be investigated in a separate study to validate their significance. Because the estimation of perfusion $(F)$ is theoretically independent of leakage, ${ }^{13}$ varying contributions of the 2 relaxation effects postextravasation would directly influence the magnitude of the resulting EF value. Fourth, previous studies in patients with glioblastoma have indicated a nonlinear relationship between the "true" $K^{\text {trans }}$ obtained from DCE-MR imaging compared with that obtained by DSC-MR imaging, ${ }^{13,30}$ which is probably due to the mixed contributions from the 2 relaxation effects in DSC-MR imaging as described above. A previous study showed that the microvessel area indicated by histopathology showed moderate correlation with the phase-derived vascular input function obtained from DCE-MR imaging and non-normalized blood volume from DSC-MR imaging ${ }^{31}$; but this correlation was not tested with the new processing method. Therefore, the microvascular permeability as derived from DSC-MR imaging should be validated against more established DCE-MR imaging-based approaches, and further studies are warranted before application of the new processing method in a clinical setting.

\section{CONCLUSIONS}

By means of a novel analysis approach, estimates of combined perfusion- and vascular permeability-related metrics were obtained from a single DSC-MR imaging acquisition. The contrast agent extraction fraction derived by this method showed improved diagnostic performance in differentiating hypervascular PCNSL from glioblastoma compared with the conventional CBVbased analysis. The analysis approach can be achieved with a single dose of gadolinium-based contrast and can be implemented in a clinical setting.

Disclosures: Atle Bjørnerud—UNRELATED: Consultancy: NordicNeuroLab AS, Comments: software development; Patents (Planned, Pending or Issued): Oslo University Hospital, Comments: patent applications relating to perfusion analysis methods*; Royalties: NordicNeuroLab AS, Comments: royalties from patents*; Stock/Stock Options: NordicNeuroLab AS. *Money paid to the institution.

\section{REFERENCES}

1. Hartmann M, Heiland S, Harting I, et al. Distinguishing of primary cerebral lymphoma from high-grade glioma with perfusionweighted magnetic resonance imaging. Neurosci Lett 2003;338: 119-22 CrossRef Medline

2. Lee IH, Kim ST, Kim HJ, et al. Analysis of perfusion weighted image of CNS lymphoma. Eur J Radiol 2010;76:48-51 CrossRef Medline

3. Xu W, Wang Q, Shao A, et al. The performance of MR perfusionweighted imaging for the differentiation of high-grade glioma from primary central nervous system lymphoma: a systematic review and meta-analysis. PLoS One 2017;12:e173430 CrossRef Medline

4. Liang R, Li M, Wang X, et al. Role of rCBV values derived from dynamic susceptibility contrast-enhanced magnetic resonance imaging in differentiating CNS lymphoma from high grade glioma: a meta-analysis. Int J Clin Exp Med 2014;7:5573-77 Medline Medline

5. Kremer S, Grand S, Remy C, et al. Cerebral blood volume mapping by MR imaging in the initial evaluation of brain tumors. J Neuroradiol 2002;29:105-13 Medline

6. Lu S, Gao Q, Yu J, et al. Utility of dynamic contrast-enhanced magnetic resonance imaging for differentiating glioblastoma, primary central nervous system lymphoma and brain metastatic tumor. Eur J Radiol 2016;85:1722-27 CrossRef Medline

7. Kickingereder P, Sahm F, Wiestler B, et al. Evaluation of microvascular permeability with dynamic contrast-enhanced MRI for the differentiation of primary CNS lymphoma and glioblastoma: radiologic-pathologic correlation. AJNR Am J Neuroradiol 2014;35: 1503-08 CrossRef Medline

8. Koeller KK, Smirniotopoulos JG, Jones RV. Primary central nervous system lymphoma: radiologic-pathologic correlation. Radiographics 1997;17:1497-526 CrossRef Medline

9. Zaharchuk G. Theoretical basis of hemodynamic MR imaging techniques to measure cerebral blood volume, cerebral blood flow, and permeability. AJNR Am J Neuroradiol 2007;28:1850-58 CrossRef Medline

10. Boxerman J, Schmainda K, Weisskoff R. Relative cerebral blood volume maps corrected for contrast agent extravasation significantly correlate with glioma tumor grade, whereas uncorrected maps do not. AJNR Am J Neuroradiol 2006;27:859-67 Medline

11. Dandois V, De Coene $B$, Laloux $P$, et al. Increased relative cerebral blood volume (rCBV) in brain lymphoma. J Neuroradiol 2011;38: 191-93 CrossRef Medline

12. Sugahara T, Korogi Y, Shigematsu Y, et al. Perfusion-sensitive MRI of cerebral lymphomas: a preliminary report. J Comput Assist Tomogr 1999;23:232-37 CrossRef Medline

13. Bjørnerud A, Sorensen AG, Mouridsen K, et al. T1- and T2*-dominant extravasation correction in DSC-MRI: part I, theoretical considerations and implications for assessment of tumor hemodynamic properties. J Cereb Blood Flow Metab 2011;31:2041-53 CrossRef Medline

14. Bjørnerud A, Emblem K. Estimation of contrast agent extravasation from the tissue residue function: application to tumor perfusion imaging. In: Proceedings of the Scientific Meeting and Exhibition of the International Society of Magnetic Resonance in Medicine, Honolulu, Hawaii. April 18-24, 2009

15. Emblem KE, Bjornerud A, Mouridsen $K$, et al. T(1)- and $\mathbf{T}(2)\left(^{*}\right)$ dominant extravasation correction in DSC-MRI: part II-predicting patient outcome after a single dose of cediranib in recurrent glioblastoma patients. J Cereb Blood Flow Metab 2011;31:2054-64 CrossRef Medline

16. Weisskoff RM BJ, Sorensen AG, Kulke ST, et al. Simultaneous blood volume and permeability mapping using a single Gd-based contrast injection. In: Proceedings of the Annual Meeting of the Society of Magnetic Resonance, San Francisco, California. August 6-12, 1994

17. Sourbron S, Ingrisch M, Siefert A, et al. Quantification of cerebral blood flow, cerebral blood volume, and blood-brain-barrier leakage with DCE-MRI. Magn Reson Med 2009;62:205-17 CrossRef Medline

18. Park SH, Goo JM, Jo CH. Receiver operating characteristic (ROC) curve: practical review for radiologists. Korean J Radiol 2004;5: 11-18 CrossRef Medline

19. Molnár PP, O’Neill BP, Scheithauer BW, et al. The blood-brain barrier in primary CNS lymphomas: ultrastructural evidence of endothelial cell death. Neuro Oncol 1999;1:89-100 Medline

20. Jain R. Perfusion CT imaging of brain tumors: an overview. AJNR Am J Neuroradiol 2011;32:1570-77 CrossRef Medline

21. Tofts PS, Brix G, Buckley DL, et al. Estimating kinetic parameters from dynamic contrast-enhanced T(1)-weighted MRI of a diffus- 
able tracer: standardized quantities and symbols. J Magn Reson Imaging 1999;10:223-32 CrossRef Medline

22. Boxerman JL, Paulson ES, Prah MA, et al. The effect of pulse sequence parameters and contrast agent dose on percentage signal recovery in DSC-MRI: implications for clinical applications. AJNR Am J Neuroradiol 2013;34:1364-69 CrossRef Medline

23. Paulson ES, Schmainda KM. Comparison of dynamic susceptibilityweighted contrast-enhanced MR methods: recommendations for measuring relative cerebral blood volume in brain tumors. Radiology 2008;249:601-13 CrossRef Medline

24. Levin JM, Kaufman MJ, Ross MH, et al. Sequential dynamic susceptibility contrast MR experiments in human brain: residual contrast agent effect, steady state, and hemodynamic perturbation. Magn Reson Med 1995;34:655-63 CrossRef Medline

25. Schramm P, Xyda A, Klotz E, et al. Dynamic CT perfusion imaging of intra-axial brain tumours: differentiation of high-grade gliomas from primary CNS lymphomas. Eur Radiol 2010;20: 2482-90 CrossRef Medline

26. Onishi S, Kajiwara Y, Takayasu T, et al. NIMG-2: analysis of perfusion CT parameters for differentiating among glioblastoma, pri- mary central nervous system lymphoma and brain metastasis. Neuro-Oncology 2017;19:vi146 CrossRef

27. Ulmer S, Liess C, Otto N, et al. The mean transit time (MTT) reflects the amount of blood brain barrier breakdown due to microvascular proliferation in glioblastoma demonstrated by perfusion weighted imaging (PWI).

28. Tietze A, Mouridsen K, Lassen-Ramshad Y, et al. Perfusion MRI derived indices of microvascular shunting and flow control correlate with tumor grade and outcome in patients with cerebral glioma. PLoS One 2015;10:e0123044 CrossRef Medline

29. Mouridsen K, Hansen MB, Østergaard L, et al. Reliable estimation of capillary transit time distributions using DSC-MRI. J Cereb Blood Flow Metab 2014;34:1511-21 CrossRef Medline

30. Skinner JT, Moots PL, Ayers GD, et al. On the Use of DSC-MRI for measuring vascular permeability. AJNR Am J Neuroradiol 2016;37: 80-87 CrossRef Medline

31. Nguyen T, Cron G, Bezzina K, et al. Correlation of tumor immunohistochemistry with dynamic contrast-enhanced and DSC-MRI parameters in patients with gliomas. AJNR Am J Neuroradiol 2016;37: 2217-23 CrossRef Medline 